\title{
Molecular Docking and 3D-QSAR Based Design of Novel Imidazopyridinone Derivatives as Pseudomonas Aeruginosa Thymidylate Kinase Inhibitors
}

\author{
S. VIKRAM KUMAR GOUD, P. SAI KIRAN REDDY, \\ S. SREE KANTH and M. VIJJULATHA*
}

Molecular Modeling and Medicinal Chemistry Group, Department of Chemistry, Nizam College, Osmania University, Basheerbagh, Hyderabad-500 001, India

vijjulathamanga@gmail.com

Received 22 August 2013 / Accepted 4 October 2013

\begin{abstract}
Thymidylate kinase (TMK) is a potential chemotherapeutic target since it is directly involved in the synthesis of deoxythymidine 5'-triphosphate an essential component in DNA replication. Inhibiting the function of TMK blocks DNA synthesis in replicating organisms. We report 3D-QSAR analysis on a series of thymidine mimetics exhibiting potent inhibitory activity against TMK. Molecular docking, Comparative molecular field analysis (CoMFA) and comparative similarity indices analysis (CoMSIA) were carried out to determine the requisite 3D structural features required for potent thymidylate kinase inhibitory activity. The molecules were divided into training set and test set, a PLS analysis was performed and QSAR models were generated. The model showed good statistical reliability which is evident from the $\mathrm{q}^{2}{ }_{\text {loo }}, \mathrm{r}_{\text {ncv }}^{2}$ and $\mathrm{r}_{\text {pred. The models }}^{2}$ were graphically interpreted using CoMFA and CoMSIA contour maps. The results obtained from this study were used for rational design of potent inhibitors against thymidylate kinase.
\end{abstract}

Keywords: Thymidylate kinase, Thymidine triphosphate, Thymidine mimetics, Molecular docking, Comparative molecular field analysis, Comparative similarity indices analysis

\section{Introduction}

New antibacterial therapeutics that utilizes novel mechanism of action is urgently needed to combat growing resistance to existing antibacterial agents for both gram-positive and gramnegative infections. Although the discovery of new antibacterial classes is extraordinarily difficult $^{1}$, need is especially high for gram negative organisms prevalent, in hospitals and in particular for infections caused by Pseudomonas aeruginosa.

Pseudomonas aeruginosa is a gram negative bacterium and an opportunistic human pathogen. It mainly targets the immuno-compromised patients and typically infects the pulmonary tract, urinary tract and even causes blood infections. P.aeruginosa is highly 
resistant to a wide range of antibiotics and disinfectants ${ }^{2}$. This pathogen has been reported to have poorer outer membrane permeability to small molecules ${ }^{3}$, for which treatment options are often limited ${ }^{4-6}$.

Thymidylate kinase (TMK) has emerged as an attractive therapeutic target because inhibiting TMK functions blocks DNA synthesis in replicating organisms ${ }^{7}$. Thymidylate kinase (TMK, EC:2.7.4.9, ATP: phosphotransferase) belongs to the nucleoside monophosphate kinase (NMPK) family and catalyzes the reversible phosphorylation of dTMP to deoxythymidine 5'-diphosphate (dTDP) in the presence of ATP as its preferred phosphoryl donor $^{8}$, situated at the junction of the de novo and salvage pathways for synthesis of deoxythymidine 5'-triphosphate (dTTP). TMK is the last specific enzyme in these pathways, therefore being essential for DNA replication \& cell growth.

Biochemical and structural characterization of PaTMK has revealed subtle differences compared to the corresponding human isozyme ( $21 \%$ of sequence identity $)^{9}$, it represents an attractive target for selectively inhibiting Pseudomonas aeruginosa DNA synthesis. Necessity for development of more potent and specific PaTMK inhibitors is an important task due to development of resistance by the organism against existing inhibitors. Recently Jun yong choi et al. ${ }^{10}$, reported imidazopyridinones as inhibitors of Pseudomonas aeruginosa targeting PaTMK, the inhibitors showed good hydrogen bond interactions with Thr101, Tyr104, Gln105 and Arg74. In an effort to design novel inhibitors of PaTMK several computational approaches are employed in development \& optimization of inhibitors. In present article we report receptor based 3D-QSAR studies using CoMFA ${ }^{11,12}$ and CoMSIA ${ }^{13}$ methodologies on imidazopyridinones derivatives. PLS ${ }^{14}$ based statistical analysis was carried out on 40 molecules to identify the correlation. The contour maps generated enabled us to explain the observed variation in activity and guided us to design new molecules.

\section{Methodology}

A total of 40 molecules were available with reported $\mathrm{IC}_{50}$ values for inhibition of paTMK ${ }^{10}$ and these values were converted to corresponding $\mathrm{pIC}_{50}$ values (Table 1). The data set was divided into training and test set of 30 and 10 molecules respectively. All molecular modeling calculations were performed on a linux operating system. The crystal structure of PaTMK bound with 1-methyl-6-benzamido-imidazopyridinone inhibitor (PDB Id: 3UWO) ${ }^{10}$ was downloaded from protein data bank. GLIDE 5.6 $6^{15}$ was used for molecular docking, protein was prepared using protein preparation module applying the default parameters and a receptor grid was generated around active site of PaTMK by selecting the cocrystallized ligand with receptor van der Waals ${ }^{16}$ scaling for non-polar atoms as 0.9 . Molecules were built using maestro build panel and prepared by LigPrep application. Structures of molecules and their $\mathrm{IC}_{50}$ and $\mathrm{pIC}_{50}$ are given in Table 1. LigPrep produces low energy conformer of the ligand using the MMFF94s force field. These molecules were docked into the grid generated from TMK protein structures using standard precision docking mode ${ }^{16}$. Cocrystallised ligand was also docked and its root mean square deviation (RMSD) was calculated to validate the docking process. Dock pose of each ligand docked into the protein was analyzed for their hydrogen bond interactions with the receptor. Analysis of dock poses of all molecules showed similar hydrogen bond interactions with Arg74, Thr101, Tyr104 and Gln105 of active site residues.

Overlay of the most promising poses (best glide score along with most physiologically similar positions) has been taken directly into 3D-QSAR analysis in this way QSAR is taken a step beyond what is usually done in such analysis. Overlay of dock pose of each ligand is shown in Figure 1. 
Table 1. Structure of PaTMK inhibitors along with $\mathrm{IC}_{50}, \mathrm{pIC}_{50}$, predicted $\mathrm{pIC}_{50}$ and Dock Score

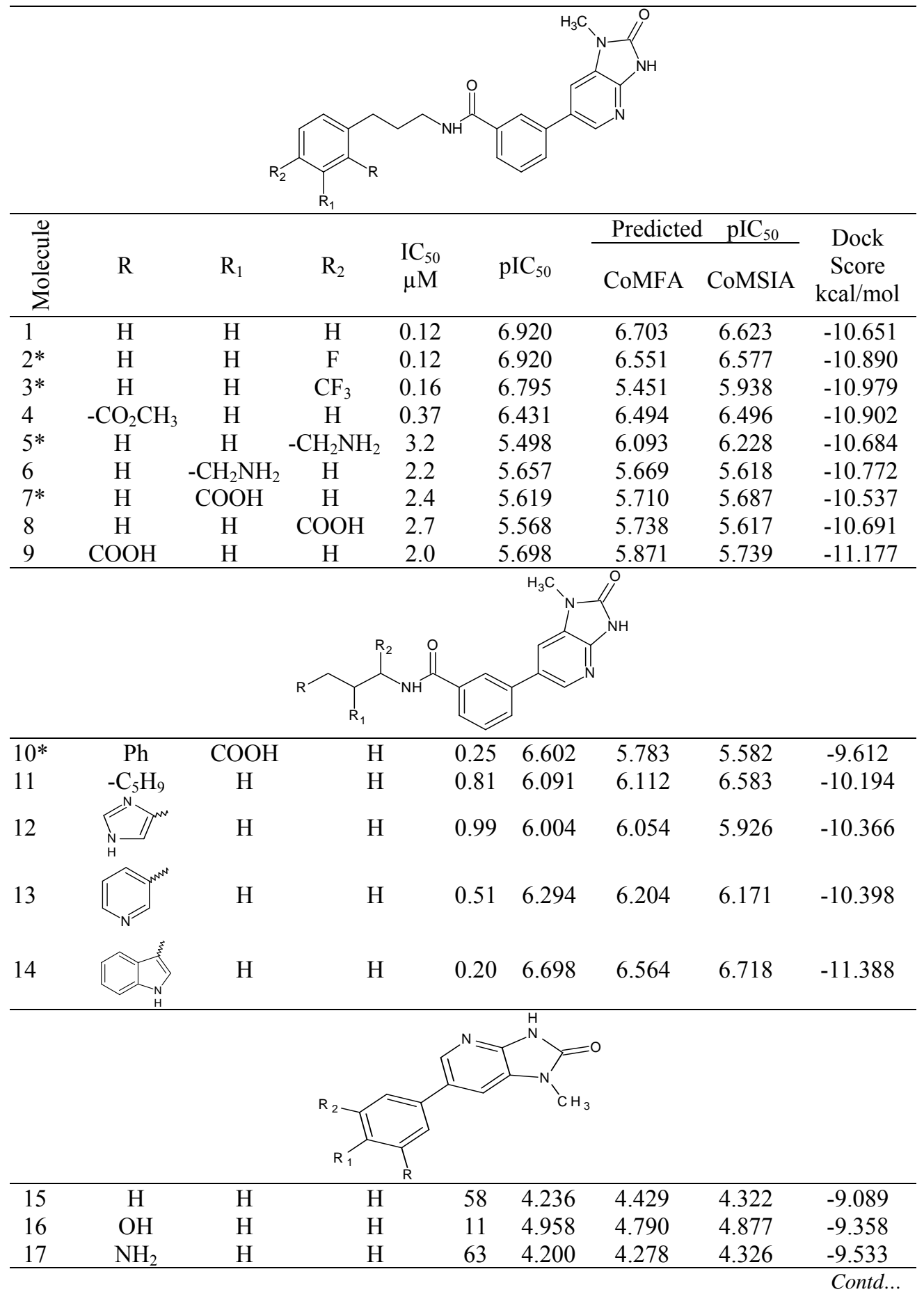




\begin{tabular}{|c|c|c|c|c|c|c|}
\hline 18 & $\mathrm{CH}_{2} \mathrm{OH}$ & $\mathrm{H}$ & 4.431 & 4.231 & 4.336 & -9.652 \\
\hline 19 & $\mathrm{H}$ & $\mathrm{C}(\mathrm{O}) \mathrm{N}\left(\mathrm{CH}_{3}\right)_{2}$ & $104 \quad 3.982$ & 4.101 & 4.142 & -9.913 \\
\hline 20 & $\mathrm{H}$ & $-\mathrm{NHC}(\mathrm{O}) \mathrm{CH}_{3}$ & 5.468 & 5.631 & 5.563 & -10.148 \\
\hline 21 & $\mathrm{H}$ & $-\mathrm{C}(\mathrm{O}) \mathrm{CH}_{3}$ & 4.886 & 4.970 & 4.496 & -10.037 \\
\hline 22 & $\mathrm{OH}$ & $\mathrm{H}$ & 4.677 & 4.754 & 4.734 & -10.278 \\
\hline 23 & $-\mathrm{CH}_{2} \mathrm{NH}_{2}$ & $\mathrm{H}$ & 5.309 & 4.806 & 5.296 & -9.642 \\
\hline $24^{*}$ & $-\mathrm{CH}_{3}$ & 2.2 & 5.657 & 5.027 & 5.488 & -10.238 \\
\hline 25 & $-\mathrm{CH}_{2} \mathrm{C}(\mathrm{O}) \mathrm{NH}_{2}$ & 0.88 & 6.055 & 6.119 & 5.879 & -10.463 \\
\hline $26^{*}$ & $-\mathrm{CH}_{2} \mathrm{C}(\mathrm{O}) \mathrm{OH}$ & 0.88 & 6.055 & 5.579 & 5.698 & -10.510 \\
\hline 27 & $-\mathrm{CH}_{2} \mathrm{CH}_{2} \mathrm{~S}(\mathrm{O})_{2} \mathrm{OH}$ & 1.3 & 5.886 & 5.936 & 5.873 & -10.125 \\
\hline 28 & $-\mathrm{CH}_{2} \mathrm{CN}$ & 2.1 & 5.677 & 5.552 & 5.605 & -10.322 \\
\hline 29 & & 2.3 & 5.638 & 5.578 & 5.498 & -9.918 \\
\hline 30 & $-\mathrm{CH}_{2} \mathrm{CH}_{2} \mathrm{C}(\mathrm{O}) \mathrm{OH}$ & 2.6 & 5.585 & 5.770 & 5.634 & -10.865 \\
\hline 31 & $-\mathrm{CH}_{2} \mathrm{CH}_{2} \mathrm{OH}$ & 2.6 & 5.494 & 5.656 & 5.444 & -10.436 \\
\hline 32 & $-\mathrm{CH}_{2} \mathrm{CH}_{2} \mathrm{~N}^{+} \mathrm{H}_{3}$ & 3.2 & 5.850 & 5.671 & 5.818 & -9.670 \\
\hline 33 & $-\left(\mathrm{CH}_{3}\right) \mathrm{CHCOOH}$ & 5.0 & 5.301 & 5.176 & 5.360 & -10.168 \\
\hline 34 & & 2.0 & 5.698 & 5.838 & 5.795 & -10.105 \\
\hline $35^{*}$ & $-\mathrm{CH}_{2} \mathrm{CH}$ & 2.9 & 5.537 & 5.389 & 4.968 & -9.843 \\
\hline $36^{*}$ & & 3.0 & 5.522 & 5.686 & 5.980 & -11.023 \\
\hline 37 & & 6.2 & 5.207 & 5.142 & 5.327 & -10.127 \\
\hline 38 & & 68 & 4.167 & 4.088 & 4.103 & -9.282 \\
\hline $39 *$ & & 66 & 4.180 & 4.535 & 4.210 & -9.690 \\
\hline 40 & & 152 & 3.818 & 3.972 & 3.981 & -9.155 \\
\hline
\end{tabular}




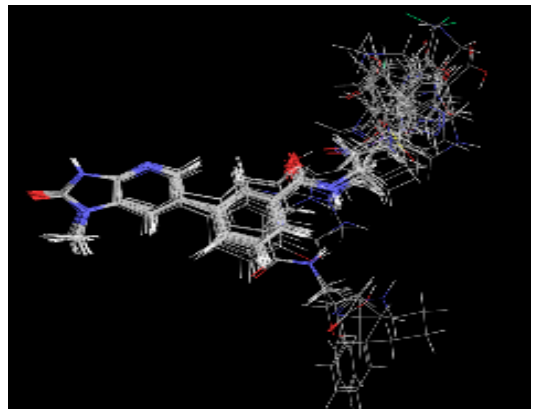

Figure 1. Dockpose alignment of TMK inhibitors

Molecules were imported into SYBYLX-1.2 molecular modeling program package ${ }^{17}$, Gasteiger-Huckel ${ }^{18}$ charges were assigned. Standard Tripos force fields were employed for

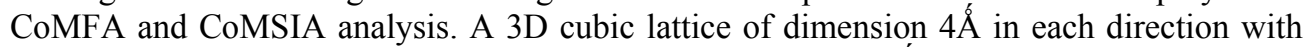
each lattice intersection of regularly spaced grid of $2.0 \AA$ was created. Steric and electrostatic parameters were calculated in CoMFA fields while hydrophobic, acceptor and donor parameters in addition to steric and electrostatic were calculated in CoMSIA fields at each lattice intersection of regularly spaced grid of $2.0 \AA$ was created. The sp 3 carbon atom was used as a probe atom to generate steric (Lennard-jones potential) field energies and a charge of +1 to generate electrostatic (columbic potential) field energies. A distance dependent dielectric constant of 1.00 was used. Steric and electrostatic fields were truncated at $+30.00 \mathrm{kcal} / \mathrm{mol}$. The similarity indices descriptors were calculated using the same lattice box employed for CoMFA calculations using sp3 carbon as a probe atom with a +1 charge,+1 hydrophobicity $\&+1$ hydrogen bond donor and +1 hydrogen bond acceptor properties.

A PLS regression was used to generate a linear relationship that correlates changes in the computed fields with changes in corresponding experimental values of biological activity $\left(\mathrm{pIC}_{50}\right)$ for the data set of ligands. Forty molecules were divided into training and test set. Biological activity values of ligands were used as dependent variables in PLS statistical analysis. Column filtering value(s) was set to $2.0 \mathrm{kcal} / \mathrm{mol}$ to improve the signalto-noise ratio by omitting those lattice points whose energy variations were below the threshold. Cross-validations were performed by leave -one -out [LOO] procedure to determine the optimal number of components (ONC) and coefficient $\mathrm{q}_{100}^{2}$. ONC obtained are then used to derive the final QSAR model using all the training set compounds with noncross validation and to obtain conventional correlation coefficient $\left(\mathrm{r}^{2}\right.$ ncv). To validate CoMFA and CoMSIA derived models, the predictive ability for the test set of compounds (expressed as $\mathrm{r}_{\text {pred }}^{2}$ ) was determined by using the following equation.

$$
\mathrm{r}_{\text {pred }}^{2}=(\mathrm{SD}-\mathrm{PRESS}) / \mathrm{SD}
$$

$\mathrm{SD}$ is the sum of squared deviations between biological activities of test set molecules and mean activity of the training set. PRESS is sum of squared deviation between observed \& predictive activities of the test set molecules. Since the statistical parameters were found to be the best for further predictions of the designed molecules. The designed molecules were also constructed, minimized and docked into the protein active site, as mentioned above.

\section{Results and Discussion}

TMK inhibitors, shown in Table 1 along with $\mathrm{IC}_{50}$ and $\mathrm{pIC}_{50}$ values, were docked into the active site; they showed five hydrogen bond interactions with the active site residues. Accuracy of docking protocol was evaluated by redocking the crystal structure ligand and its 
RMSD from the experimental binding mode determined by x-ray crystallography was calculated, and it gave a value of $0.4803 \AA$ (Figure 2). The correlation between dock score (glide score) and $\mathrm{pIC}_{50}$ gave a correlation coefficient value (r) of 0.693 , which shows appreciable relation between biological activity and docking, scatter plot of $\mathrm{pIC}_{50}$ and glide score is shown in Figure 3.

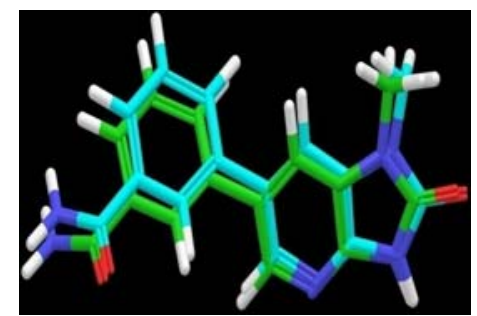

Figure 2. Superimposition of crystal structure pose (cyan) on docked pose (green) of cocrystallized ligand

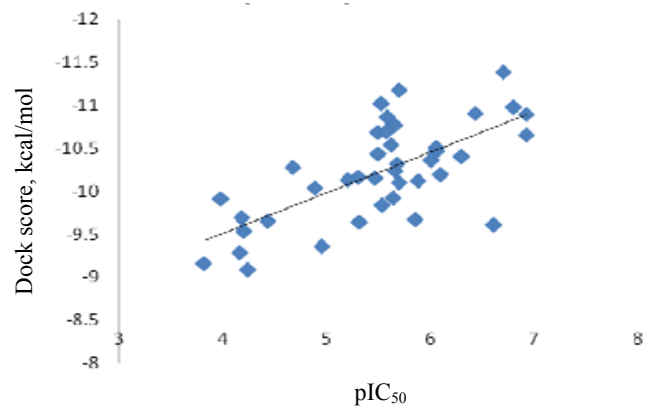

Figure 3. Scatter Plot of $\mathrm{pIC}_{50}$ vs. Dock Score

Molecules having specific hydrogen bond interactions with active site of the receptor, where imidazopyridinone $\mathrm{C}=\mathrm{O}$ act as hydrogen bond acceptor to $\operatorname{Arg} 74$, Thr101, while both $\mathrm{NH}$ of cyclic imidazopyridinone ring act as donor and acceptor to $\mathrm{C}=\mathrm{O}$ group of $\mathrm{Gln} 105$ and benzamido $\mathrm{C}=\mathrm{O}$ act as hydrogen bond acceptor to $-\mathrm{OH}$ of Tyr104 in TMK, were considered. The 3D-QSAR, CoMFA and CoMSIA analysis were carried out in the reported inhibitors (Table 1). Molecules having precise $\mathrm{IC}_{50}$ values were selected and those that did not show interactions with the protein active site (via docking) were removed from the data set.

A set of 40 molecules were used for derivation of model, these were divided into a training set of 30 molecules \& test set of 10 , keeping in view that the activity range is with a minimum of $3 \log$ units differences in both the sets. CoMFA\& CoMSIA statistical analysis is summarized in Table 2. Statistical data shows $\mathrm{q}_{\text {loo }}^{2} 0.577$ for CoMFA 0.670 for CoMSIA models, $\mathrm{r}_{\text {ncv }}^{2}$ of 0.961 and 0.962 for CoMFA \& CoMSIA, respectively, which indicates a good internal predictive ability of models. To test the predictive ability of models, a test set of 10 molecules exchanged from the model derivation was used. The predictive correlation coefficient $r_{\text {pred }}^{2}$ of 0.521 for CoMFA and 0.603 for the CoMSIA models indicate good external predictive ability for the models. Scatter plot for actual and predicted $\mathrm{pIC}_{50}$ values for CoMFA and CoMSIA studies shown in Figure 4. The CoMSIA model showed better results than CoMFA model, this shows that the hydrophilic and hydrophobic fields which were not included in the CoMFA model are important for explaining the potency of the molecules. This is also evident from the docking results. The predicted activity and glide scores of the molecules are provided in Table 1 . 
Table 2. Summary of PLS results

\begin{tabular}{ccc}
\hline Statistical parameters & CoMFA & CoMSIA \\
\hline $\mathrm{q}^{2}$ loo & 0.577 & 0.670 \\
No. of molecules in training set & 30 & 30 \\
No. of molecules in test set & 10 & 10 \\
ONC & 5 & 4 \\
SEE & 0.176 & 0.168 \\
$\mathrm{r}_{\text {ncv }}^{2}$ & 0.961 & 0.962 \\
$\mathrm{~F}_{\text {ratio }}^{2}$ & 116.750 & 159.649 \\
$\mathrm{r}_{\text {pred }}$ & 0.521 & 0.603 \\
Steric & & \\
Electrostatic & 72.8 & 20.1 \\
Hydrophobic & 27.2 & 7.8 \\
Donor & -- & 18.9 \\
Acceptor & -- & 33.3 \\
\hline
\end{tabular}

$q^{2}{ }_{\text {loo: }}$ : Cross-validated correlation coefficient by leave one out method; $r_{n c v}^{2}$ : non-cross-validated correlation coefficient; $r_{\text {pred: }}^{2}$ predictive correlation coefficient on test set; SEE: standard error of estimate; $F_{\text {ratio: }}$ Fischer test value; ONC: optimal Number of principal components
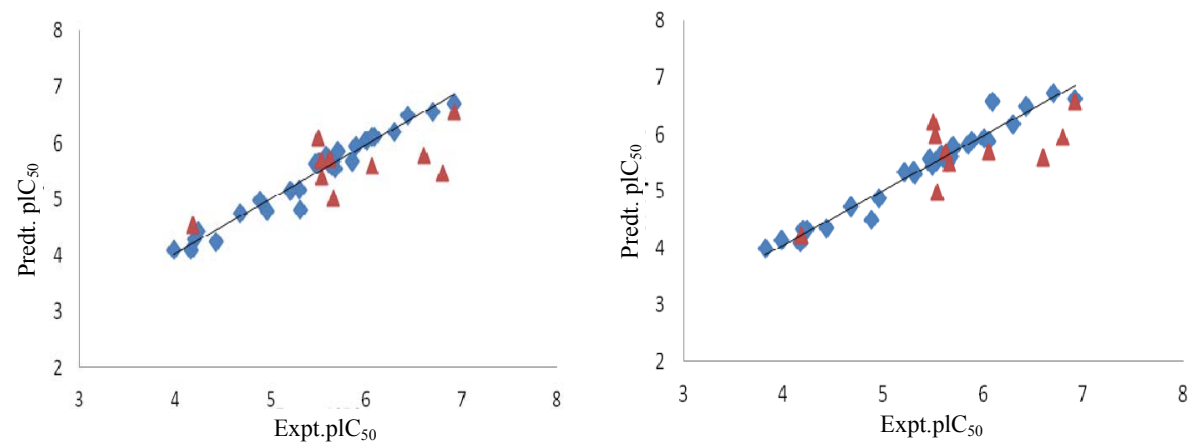

Figure 4. Graphs of the experimental versus predicted $\mathrm{pIC}_{50}$ values of the training $(\bullet)$ and test $(\boldsymbol{\Delta})$ compounds from the CoMFA and CoMSIA models

\section{CoMFA and CoMSIA contour maps}

To visualize the information content of the derived 3D-QSAR models, CoMFA and CoMSIA contour maps were generated. The Contour plots are representation of the lattice points $\&$ the difference in the molecular field values, at lattice points, strongly connected with difference in the receptor binding affinity. Molecular fields define favorable or unfavourable interaction energies of aligned molecules with a probe atom traversing across lattice plots suggests modification required to design new molecules. Contour maps of CoMFA denote region in the space where molecules would favourably or unfavourably interact with the receptor while CoMSIA contour maps denote those areas within the specified region where presence of a group with a particular physicochemical activity binds to the receptor. CoMFA and CoMSIA results were graphically interpreted by field contribution maps using "STDEV*COEFF" field type. All contours represented default 80 and $20 \%$ level contribution for favoured and disfavoured regions. 
The most potent analogue, molecule 1 was embedded in the map Figure $5(a, b)$, to demonstrate its affinity for steric and electrostatic regions of inhibitors. The areas of yellow indicate regions of steric hindrance to activity, while green areas indicate a steric contribution to potency. The blue regions indicate a positive electrostatic charge potential associated with increased activity, while red region show negative charge potential. The steric contour maps of CoMFA show, the green regions surrounding alkyl linker attached to benzene ring for substitution of sterically bulky favoured groups. The yellow contour over the benzamide ring suggests steric bulk disfavoured region in molecule 1 . Electrostatic contour maps shows two regions of blue contours above amide group and at para position of benzene ring attached to the alkyl chain for positive electrostatic potentials, a red contour is seen near the meta position of both the phenyl rings in molecule 1 suggesting substitution of more negatively charged substituents at this position will significantly improve biological activity.

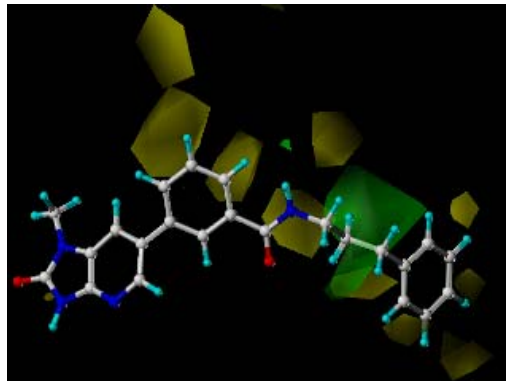

(a)

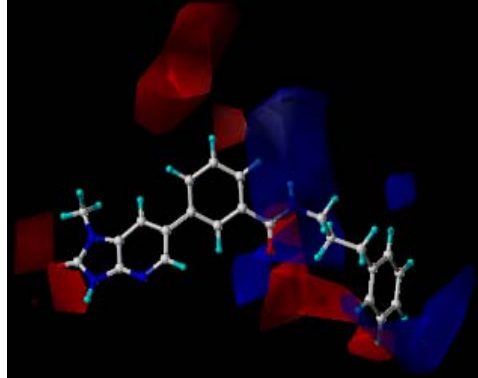

(b)

Figure 5. CoMFA steric standard deviation (S.D.* coefficient) contour maps illustrating steric (a) and electrostatic (b) features in combination with molecule 1. Green contours show favourable bulky group substitution at that point while yellow regions show disfavourable bulky group substitution for activity. Red contours indicate negative charge favouring activity, whereas blue contours indicate positive charge favouring activity

Figure 6(a-e) shows the contour maps derived from the CoMSIA PLS model. The most potent analogue, molecule 1, was embedded in the maps to demonstrate its affinity for the steric, electrostatic, hydrophobic, hydrogen bond donor and acceptor regions of inhibitors. The steric map is similar to the CoMFA steric map showing favoured and disfavoured regions. In steric contour embedded by molecule 1 the benzyl group is penetrating into the green region for bulky favoured substitution and the entire molecule is away from the disfavoured yellow region. The electrostatic contour map shows a red contour over the carbonyl of benzamide ring in molecule 1 indicating a negative electrostatic potential. The blue regions over the $-\mathrm{NH}$ of amide, $\mathrm{CH}_{2}$ attached to benzene ring indicates regions with positive electrostatic potentials in molecule 1 . The hydrophobic contours shows, meta and para positions of benzene ring incorporated into the favoured yellow region suggests substituting sterically bulky, hydrophobic groups further increases biological activity. A white contour is observed around the alkyl linker region suggesting hydrophilic substitution on the linker region will increase activity. The donor contour map show benzamide group incorporated into disfavoured purple regions in molecule 1. The acceptor contour maps shows favoured magenta region near to the carbonyl group, disfavoured red region is observed on to the nitrogen of amide and para position of benzene ring suggesting substitution with acceptor groups will increase the potency. 


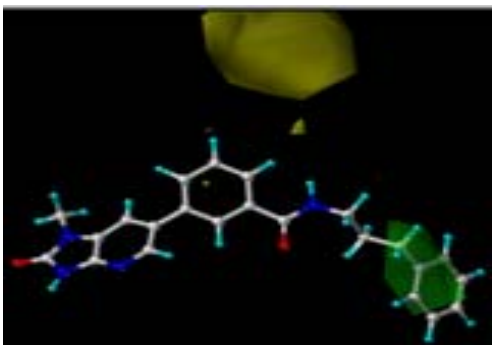

(a)

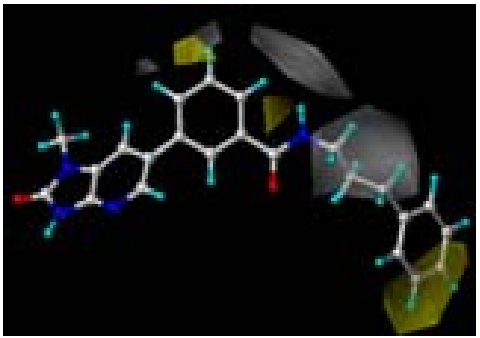

(c)

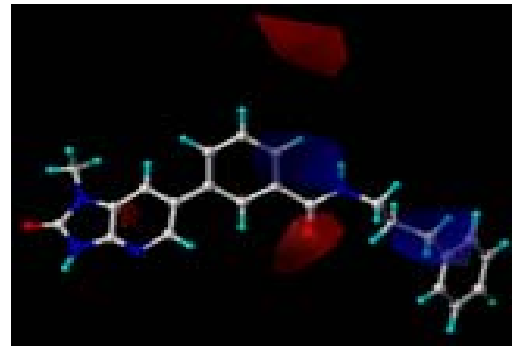

(b)

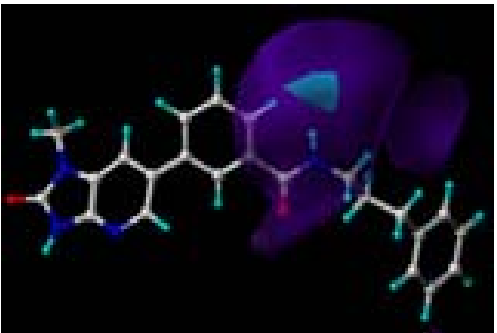

(d)

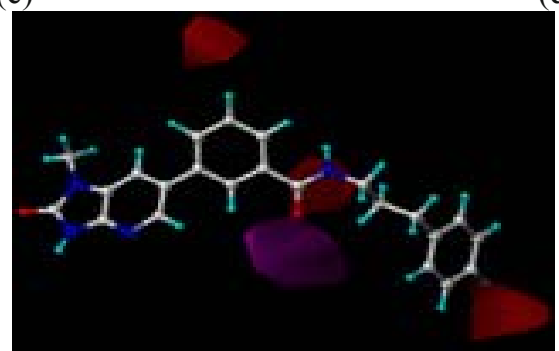

(e)

Figure 6. CoMSIA SD* coefficient contour maps in combination with molecule 1 illustrating features (a) Steric: Green contours represent favourable bulky group substitution at that point while yellow regions are disfavorable for activity; (b) Electrostatic: Red contours indicate negative charge favouring activity, whereas blue contours indicate positive charge favouring activity; (c) Hydrophobic: Yellow contour represent hydrophobic favoured region, white indicates the hydrophilic favoured regions; (d) Donor: The purple contour represents $H$-bond donor disfavoured regions while cyan indicates $H$-bond donor favoured and (e) Acceptor: Magenta and red contour represent $H$-bond acceptor disfavoured and favoured regions.

Detailed contour map analysis of both CoMFA and CoMSIA models empowered us to identify structural requirements for observed inhibitory activity (Figure 8). The molecules were modified to further improve the inhibition activity towards PaTMK. Molecule 1 was chosen as a reference structure to design molecules (Table 3) with increased potency. New molecules designed were docked into the protein active site and they showed similar interactions with comparable dock scores and dock poses were used to predict the activity by applying the 3D-QSAR model. The new molecules showed better predicted activity with respect to the most active molecule. Sterically bulky \& hydrophobic groups (ethyl, $t$-butyl, n-butyl, benzyl) substituted on $\mathrm{CH}_{2}$ of alkyl linker region attached to benzene ring and also hydrophilic groups $\left(\mathrm{OH}, \mathrm{COOH}, \mathrm{SCH}_{3}\right)$ substituted on meta position of benzene ring attached to the amide group increased the activity. 


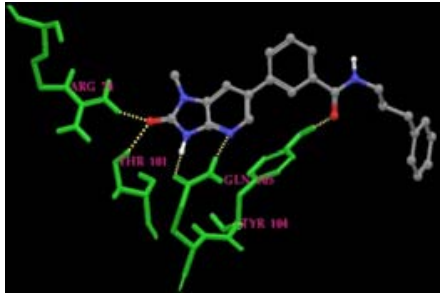

(a)

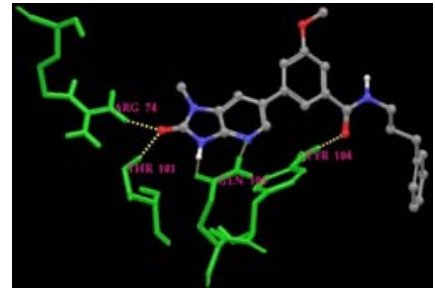

(b)

Figure 7. Docked pose of (a) molecule 1 and (b) molecule N5 in the protein active site showing similar hydrogen bond interactions with Arg 74, Thr 101, Gln 105, and Thr 104

Sterically bulky favoured and

Hydrophobic region

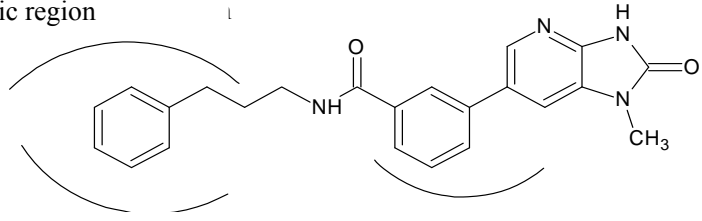

Electronegative charge favoured region, sterically bulky disfavoured region

Hydrophilic, electronegative charge favoured region

Figure 8. Structural requirements for PaTMK inhibitors obtained from CoMFA (CF) and CoMSIA (CMS) contour map analysis

Table 3. Structure, predicted pIC50 and dock score of designed molecules

\begin{tabular}{|c|c|c|c|c|c|c|c|c|c|}
\hline 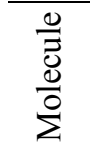 & $\mathrm{R}_{1}$ & $\mathrm{R}_{2}$ & $\mathrm{R}_{3}$ & $\mathrm{R}_{4}$ & $\mathrm{R}_{5}$ & $\mathrm{R}_{6}$ & $\begin{array}{c}\text { Pred } \\
\text { pIC }_{50} \\
\text { CoMFA }\end{array}$ & $\begin{array}{c}\text { Pred } \\
\text { pIC }_{50} \\
\text { CoMSIA }\end{array}$ & $\begin{array}{c}\text { DockScore } \\
\mathrm{kcal} / \mathrm{mol}\end{array}$ \\
\hline N1 & $\mathrm{H}$ & $\mathrm{H}$ & $\mathrm{H}$ & ethyl $(R)$ & $\mathrm{H}$ & $\mathrm{H}$ & 7.208 & 6.438 & -10.546 \\
\hline $\mathrm{N} 2$ & $\mathrm{H}$ & t-butyl & $\mathrm{H}$ & $\mathrm{H}$ & $\mathrm{H}$ & $\mathrm{H}$ & 6.521 & 6.965 & -10.576 \\
\hline N3 & $\mathrm{Cl}$ & $\mathrm{H}$ & $\mathrm{H}$ & $\mathrm{H}$ & $-\mathrm{S}\left(\mathrm{CH}_{3}\right)$ & $\mathrm{H}$ & 7.139 & 5.815 & -10.825 \\
\hline N4 & $\mathrm{OH}$ & $\mathrm{H}$ & $\mathrm{H}$ & $\mathrm{H}$ & $-\mathrm{S}\left(\mathrm{CH}_{3}\right)$ & $\mathrm{H}$ & 7.377 & 5.951 & -10.911 \\
\hline N5 & $\mathrm{H}$ & $\mathrm{H}$ & $\mathrm{H}$ & $\mathrm{H}$ & $\mathrm{H}$ & $\mathrm{OH}$ & 7.113 & 6.673 & -10.989 \\
\hline N6 & $\mathrm{H}$ & $\mathrm{H}$ & $\mathrm{H}$ & $\mathrm{H}$ & $\mathrm{H}$ & $\mathrm{COOH}$ & 6.903 & 7.090 & -10.645 \\
\hline N7 & $\mathrm{H}$ & $\mathrm{H}$ & $-\mathrm{CH}_{2} \mathrm{Ph}$ & $-\mathrm{CH}_{2} \mathrm{Ph}$ & $\mathrm{H}$ & $\mathrm{H}$ & 6.736 & 7.119 & -11.476 \\
\hline N8 & $\mathrm{H}$ & $\mathrm{H}$ & $\mathrm{H}$ & $\mathrm{H}$ & $\mathrm{H}$ & $-\mathrm{O}(\mathrm{pr})$ & 7.567 & 6.645 & -10.455 \\
\hline N9 & n-butyl & $\mathrm{H}$ & $\mathrm{H}$ & ethyl $(R)$ & $\mathrm{H}$ & $-\mathrm{S}\left(\mathrm{CH}_{3}\right)$ & 6.967 & 6.535 & -9.365 \\
\hline N10 & $-\mathrm{CHCl}_{2}$ & $\mathrm{H}$ & $\mathrm{H}$ & $\mathrm{H}$ & $-\mathrm{S}\left(\mathrm{CH}_{3}\right)$ & $\mathrm{H}$ & 7.102 & 5.629 & -10.851 \\
\hline N11 & $\mathrm{H}$ & $\mathrm{H}$ & $\mathrm{H}$ & $\mathrm{H}$ & $-\mathrm{S}\left(\mathrm{CH}_{3}\right)$ & $\mathrm{H}$ & 7.311 & 5.783 & -10.695 \\
\hline N12 & $\mathrm{F}$ & $\mathrm{H}$ & $\mathrm{H}$ & $\mathrm{H}$ & $-\mathrm{S}\left(\mathrm{CH}_{3}\right)$ & $\mathrm{H}$ & 7.275 & 5.795 & -11.183 \\
\hline N13 & $\mathrm{H}$ & $\mathrm{H}$ & $\mathrm{H}$ & n-butyl $(R)$ & $\mathrm{H}$ & $\mathrm{OH}$ & 7.452 & 6.799 & -10.851 \\
\hline N14 & ethyl & $\mathrm{H}$ & $\mathrm{H}$ & Ethyl $(R)$ & $\mathrm{H}$ & $\mathrm{OH}$ & 7.062 & 6.727 & -10.395 \\
\hline
\end{tabular}




\section{Conclusion}

Molecular docking based 3D-QSAR studies are widely used tools for understanding binding modes of the molecule to the protein receptors and rationalize structural requirements for the inhibitory activity of the molecules. CoMFA \& CoMSIA methodologies were used to build models for PaTMK inhibitory activity of imidazopyridinone derivatives. The generated models have statistical reliability that is evident from high $\mathrm{r}^{2}$ and $\mathrm{q}^{2}$ values for all the models. Based on detailed contour map analysis improvement in PaTMK binding affinity can be achieved through conformationally restricted substitution on $\mathrm{CH}_{2}$ of alkyl linker region attached to benzene ring and meta position of benzene ring attached to the amide group. The designed molecules based on these parameters showed better predictive activity than reference molecule, this indicates QSAR models generated have good predictive ability to design potent inhibitors. These molecules can be synthesized to generate a greater number of molecules with required pharmacokinetics for further clinical studies.

\section{Acknowledgement}

We gratefully acknowledge support for this research from Department of Science and Technology, New Delhi, India, University Grants Commission, New Delhi, India and Department of chemistry, Nizam College, Hyderabad, India. We also acknowledge Schrödinger Inc. for GLIDE software, Tripos Inc. for SYBYLX-1.2.

\section{References}

1. Payne D J, Gwynn M N, Holmes D J and Pompliano D L, Nat Rev Drug Dis., 2007, 6(1), 29; DOI:10.1038/nrd2201

2. Stover C K, Pham X Q, Erwin A L, Mizoguchi S D, Warrener P, Hickey M J, Brinkman F S, Hufnagle W O, Kowalik D J, Lagrou M, Garber R L, Goltry L, Tolentino E, Westbrock W S, Yuan Y, Brody L L, Coulter S N, Folger K R, Kas A, Larbig K, Lim R, Smith K, Spencer D, Wong G K, Wu Z, Paulsen I T, Reizer J, Saier M H, Hancock R E, Lory S and Olson M V, Nature, 2000, 406, 959.

3. Mdluli K E, Witte P R, Kline T, Barb A W, Erwin A L, Mansfield B E, McClerren A L, Pirrung M C, Tumey L N, Warrener P, Raetz C R H and Stover C K, Antimicrob Agents Chemother., 2006, 50(6), 2178-2184; DOI:10.1128/AAC.00140-06

4. Obritsch M D, Fish D N, MacLaren R and Jung R, Pharmacotherapy, 2005, 25(10), 1353-1364.

5. Rice L B, Curr Opin Microbiol., 2009, 12(5), 476-461; http://dx.doi.org/10.1016/j.mib.2009.08.001

6. Rice L B, Cleve Clin J Med., 2007, 74(4), S12; DOI:10.3949/ccjm.74.Suppl_4.S12.

7. Kandeel M, Kato A, Kitamura Y and Kitade Y, Nucleic Acids Symp Ser., 2009, 53(1), 283-284; DOI:10.1093/nass/nrp142

8. Anderson E, Nucleoside and Nucleotide Kinases. In the Enzymes $3^{\text {rd }}$ Ed., Academic Press: New York, 1973, 8, 49; ISBN-10: 0121227103

9. Munier-Lehmann H, Chafotte A, Pochet S and Labesse G, Protein Sci., 2001, 10(6), 1195-1205; DOI:10.1110/ps.45701

10. Choi J Y, Plummer M S, Starr J, Desbonnet C R, Soutter H, Chang J, Miller J R, Dillman K, Miller A A and Roush W R, J Med Chem., 2012, 55(2), 852-870; DOI:10.1021/jm201349f

11. Cramer R D, Patterson D E and Bunce J D, J Am Chem Soc., 1988, 110(18), 59595967; DOI:10.1021/ja00226a005. 
12. Cramer III R D, Patterson D E, Ildiko E. Frank and Bunce J D, Quant Struct Act Relat., 1988, 7(1), 18-25; DOI:10.1002/qsar.19880070105

13. Klebe G, Abraham U and Mietzner T, J Med Chem., 1994, 37(24), 4130-4146; DOI:10.1021/jm00050a010

14. Wold S, Johansson A and Cochi M, PLS-Partial least squares projection to latent structures. Lieden: ESCOM, 1993, 523-550.

15. Schrödinger, LLC, New York: Glide Version 5.6, 2010

16. Friesner R A, Banks J L, Murphy R B, Halgren T A, Klicic J J, Mainz D T, Repasky M P, Knoll E H, Shelley M, Perry J K, Shaw D E, Francis P and Shenkin P S, J Med Chem., 2004, 47(7), 1739-1749; DOI:10.1021/jm0306430

17. Sybyl-X1.2, Tripos Associates, St. Louis (MO), 2010.

18. Gasteiger J and Marsili M, Tetrahedron, 1980, 36(22), 3219-3228;

http://dx.doi.org/10.1016/0040-4020(80)80168-2 